\title{
Comparison of novel liquid electrodes for silica optical fiber thermal poling
}

\author{
F. De Lucia ${ }^{1}$, C. Corbari ${ }^{2}$, D. Keefer ${ }^{3}$, P. J. A. Sazio ${ }^{1^{*}}$ \\ ${ }^{I}$ Optoelectronics Research Centre, University of Southampton, SO17 1BJ, UK \\ ${ }^{2}$ Renishaw plc, New Mills, Wotton-under-Edge, Gloucestershire, GL12 8JR UK \\ ${ }^{3}$ Department of Chemistry, Pennsylvania State University, University Park, PA 16802, USA \\ *pjas@soton.ac.uk
}

\begin{abstract}
We report experimental analysis of optical fibers thermally poled over long lengths using novel types of internal liquid electrodes to generate effective second-order nonlinearities. Our analysis includes transmission losses, depletion region formation, SHG at telecom pump wavelengths.
\end{abstract}

OCIS codes: (190.4370) Nonlinear optics; (060.2310) Fiber optics; (190.2620) Harmonic generation and mixing;

\section{Introduction}

The thermal poling technique, invented by Myers et al. [1] originally using bulk glass samples, has been successfully used for a number of years with silica optical fibers [2], by freezing an electric field into the glass and thus inducing an effective second order nonlinear susceptibility into the sample due to a process of third order nonlinear optical rectification. Despite the promising perspective given by this discovery, thermally poled optical fibers are usually plagued by a number of technical issues that can limit the fabrication of long, robust, low loss and easily fabricated all-fiber devices, such as for example frequency converters based on SHG in periodically poled silica fibers (PPSF) [3]. In 2014 De Lucia et al. [4] demonstrated that it is possible to overcome issues related to the insertion and direct electrical contact to solid electrodes inside the fibers by using a novel indirect electrostatic induction poling mechanism that exploits liquid metal electrodes instead. The use of liquids not only allows for poling of very long (meter scale) samples, but more importantly, our novel choice of electrode materials allows for their facile removal after thermal poling is complete in order to minimize linear optical losses.

Here we report the behavior of different materials used as electrodes. The resulting fiber devices are characterized in terms of linear transmission losses after electrode removal. In addition they are examined for evidence of depletion region formation and for the development of second-order nonlinearities as evaluated by quasi-phase matching (QPM) at a particular pump wavelength, by measuring the generated second harmonic generated (SHG) power. Comparing electrode materials can identify new routes towards the realization of long, robust, low loss, highly efficient nonlinear all-fiber devices for use in, for example, frequency conversion [3], electro-optic modulation and switching [5] and polarization entangled photon pair generation.

\section{Silica fiber thermal poling using novel liquid electrode types}

The schematic in Fig. 1 shows the experimental setup used to pole (up to $60 \mathrm{~cm}$ long) samples using liquid electrodes.

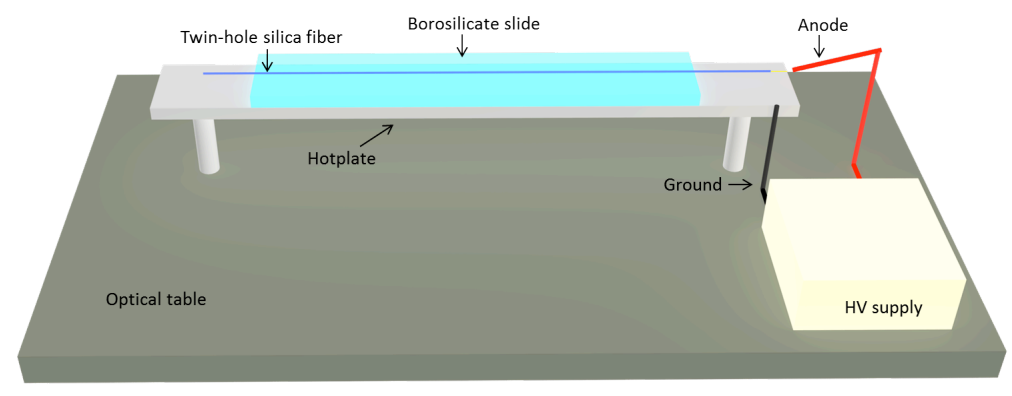

Fig. 1. Silica fiber thermal poling setup

To ensure reproducibility we used four identical samples (Acreo Fiberlab twin-hole silica fiber, OD $=125 \mu \mathrm{m}$, electrode hole diameter $=30 \mu \mathrm{m}$, Germania doped core diameter $=4 \mu \mathrm{m}$ with NA $=0.17$ as shown in Fig. 2). The electrode liquids used to fill the twin-hole fiber samples were Gallium, Mercury, $20 \% \mathrm{HCl}$ solution and ordinary tap water. The liquid metals were introduced into the samples using pressurization techniques [5] whereas the aqueous solutions could be inserted via normal capillary action. After filling, tungsten wires are inserted into the twin holes 
at one end of the samples, ensuring electrical contact with the liquid. Both ends are then sealed with epoxy or superglue and all four samples are then poled under the same experimental conditions, with an applied voltage of +5 $\mathrm{kV}$, temperature of the hotplate $=300^{\circ} \mathrm{C}$, heating time $=120$ mins, cooling time $=45$ mins .

\section{Performance comparison of electrodes}

As expected, under the large applied bias and temperature of $300^{\circ} \mathrm{C}$, the non-volatile liquid metal electrodes generated depletions regions within the fiber, visualized using HF acid decorative etching [6] as seen in Fig. 2. Remarkably, despite these extreme conditions, the aqueous solutions also yielded well-defined depletion regions throughout the entire $60 \mathrm{~cm}$ length of the test samples. This highly counterintuitive result is currently under further investigation, as it is not yet fully understood how electrical continuity is maintained during poling while the fiber is held well above the boiling points of these solutions for extended periods. As we will discuss, the losses in all samples are similar in magnitude, although typically much lower in the samples poled using aqueous solutions (see Table. 1). The dataset of the experimental data can be found at: http://dx.doi.org/10.5258/SOTON/386021.

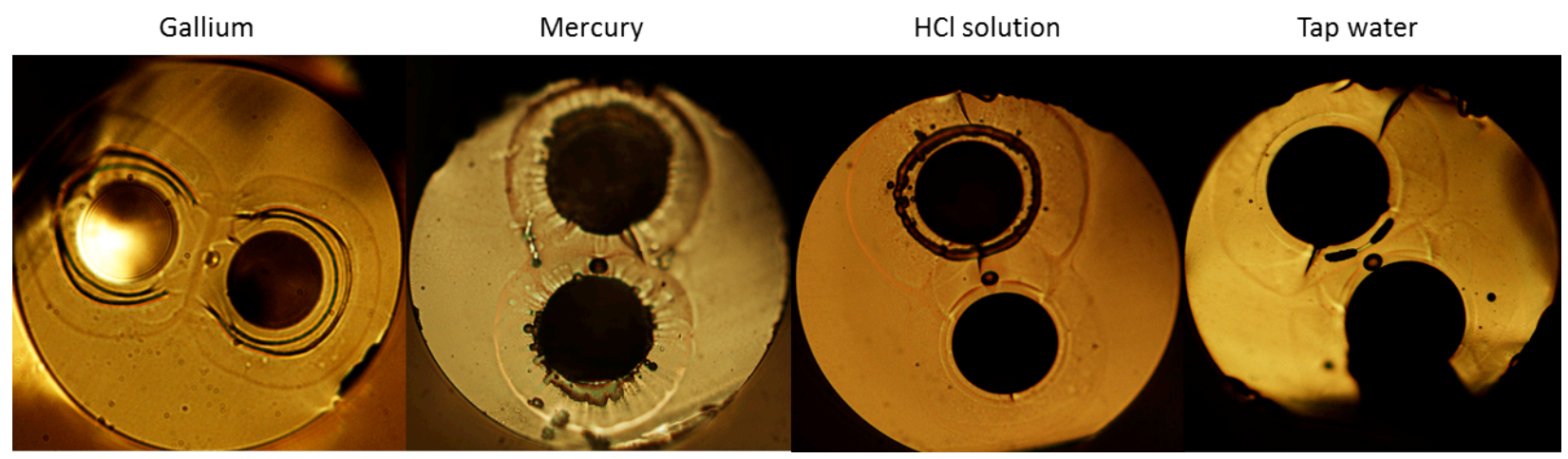

Fig. 2. Representative cross sectional optical micrographs of the HF etched samples poled using novel liquid electrode types. The decorative etching process reveals the presence of depletion regions in all four samples, usually indicative of an induced secondorder nonlinearity.

Table 1: average values of linear losses and of estimated $\chi^{(2)}$ eff for samples poled by means of different liquid electrodes

\begin{tabular}{|c|c|c|c|c|}
\hline & Gallium & Mercury & HCl solution in DI water & Tap water \\
\hline Linear losses $(\mathrm{dB}) @ 1550 \mathrm{~nm}$ & 1.74 & 1.09 & 0.59 & 0.79 \\
\hline $\mathrm{X}^{(2)}{ }_{\text {eff }}(\mathrm{pm} / \mathrm{V})$ & 0.056 & 0.08 & $<0.01$ & $<0.01$ \\
\hline
\end{tabular}

Finally, the induced $\chi^{(2)}$ nonlinearities were estimated by inscribing a QPM grating into the poled samples using the UV erasure technique [4] and measuring the resulting SHG at a pump wavelength of $1550 \mathrm{~nm}$. For this parameter, the liquid metal poled samples gave far superior performance than the aqueous (average $\chi_{\text {eff }}^{(2)}<0.01$ $\mathrm{pm} / \mathrm{V}$ for $\mathrm{H}_{2} \mathrm{O}$, compared to $0.08 \mathrm{pm} / \mathrm{V}$ for $\mathrm{Hg}$, which to our knowledge is the highest ever recorded in a silica optical fiber) and a full comparison of all sample characteristics will be presented and discussed.

\section{Acknowledgments}

The authors acknowledge EPSRC EP/I035307/1 for financial support.

\section{References}

[1] R. A. Myers, N. Mukherjee, and S. R. J. Brueck, "Large second-order nonlinearity in poled fused silica", Opt. Lett. 16, 1732-1734 (1991).

[2] W. Margulis, O. Tarasenko and N. Myrén, "Who needs a cathode? Creating a second-order nonlinearity by charging glass fiber with two anodes", Opt. Exp. 17, 15534 - 15540 (2009).

[3] C. Corbari, A. V. Gladyshev, L. Lago, M. Ibsen, Y. Hernandez, and P. G. Kazansky, "All-fiber frequency-doubled visible laser", Opt. Lett. 39, 6505-6508 (2014).

[4] F. De Lucia, D. Huang, C. Corbari, N. Healy, and P. J. A. Sazio, “Optical fiber poling by induction”, Opt. Lett. 39, 6513 - 6516 (2014).

[5] M. Fokine, L.E. Nilsson, A.A. Claesson, D. Berlemont, L. Kjellberg, L. Krummenacher and W. Margulis”, Integrated fiber Mach-Zehnder interferometer for electro-optic switching", Opt. Lett. 27, 1643 - 1645 (2002).

[6] T. G. Alley, and S. R. J. Brueck, "Visualization of the nonlinear optical space-charge region of bulk thermally poled fused-silica glass", Opt. Lett. 23, 1170 - $1172(1998)$. 